\title{
ON GORDON'S METHOD OF SOLVING DUAL INTEGRAL EQUATIONS
}

\author{
by J. BURLAK \\ (Received 18 December, 1962)
}

1. Dual integral equations of the form

$$
\begin{aligned}
& \int_{0}^{\infty} u^{\alpha} \psi(u) J_{v}(x u) d u=f(x) \quad(0 \leqq x<1), \\
& \int_{0}^{\infty} \psi(u) J_{v}(x u) d u=g(x) \quad(x>1),
\end{aligned}
$$

where $f(x)$ and $g(x)$ are given and $\psi(x)$ is the unknown, have been increasingly studied in recent years; the first solutions were given for the case $g(x) \equiv 0$ by Titchmarsh [1] (for $0<\alpha<2$ ) and Busbridge [2] (for $-2<\alpha<0$ ). An interesting and much simpler method of solving the equations in the same case, $g \equiv 0$, was given by Gordon [3]. He also showed that the problem of solving the general equations (1) and (2) can be reduced to a problem in which $g \equiv 0$. He did not pursue this idea as far as finding and simplifying the solution of (1) and (2) but this has been done recently (see [4]) and Noble [5] used a similar idea in treating the case $f \equiv 0$.

What we wish to show here is that Gordon's method of treating the case $g \equiv 0$ can be adapted to deal equally well with the case $f \equiv 0$; since (1) and (2) are linear, the solution of (1) and (2) can then be obtained by superposition of the solutions in these separate cases. This has affinities with the work of Lowengrub and Sneddon [6], who showed that an elegant method developed by Copson [7] for the case $g \equiv 0$ can be adapted to treat the case $f \equiv 0$. This superposition of solutions was first used by Noble [5]; he also [8] gave a direct but lengthy solution of the general equations (1) and (2). As in most work on the subject the analysis here is formal; for instance, no attempt is made to justify interchange of orders of integration.

2. We now consider the equations

$$
\begin{aligned}
& \int_{0}^{\infty} u^{a} \psi(u) J_{v}(x u) d u=0 \quad(0 \leqq x<1), \\
& \int_{0}^{\infty} \psi(u) J_{v}(x u) d u=g(x) \quad(x>1) .
\end{aligned}
$$

The essential idea of the method is that of transforming the equations so that they involve the same integrand: the solution can then be written down by the Hankel inversion theorem (see 
Sneddon [9]). The argument rests on two results both of which follow from the WeberSchaftheitlin discontinuous integral (Watson [10, p. 398]):

$$
\begin{aligned}
& \int_{0}^{\infty} J_{\mu}(a t) J_{\nu}(b t) t^{-\lambda} d t=\frac{b^{\nu} \Gamma\left\{\frac{1}{2}(\mu+\nu-\lambda+1)\right\}}{2^{2} a^{\nu-\lambda+1} \Gamma(v+1) \Gamma\left\{\frac{1}{2}(\lambda+\mu-v+1)\right\}} \\
& \times_{2} F_{1}\left\{\frac{1}{2}(\mu+v-\lambda+1), \frac{1}{2}(v-\lambda-\mu+1) ; \nu+1 ; b^{2} / a^{2}\right\},
\end{aligned}
$$

where $0<b<a$; a similar formula, with $a$ and $b$, and $\mu$ and $v$, interchanged, holds when $0<a<b$. The integral converges if

It follows from (5) that

$$
\operatorname{Re}(\mu+\nu+1)>\operatorname{Re}(\lambda)>-1 \text {. }
$$

provided that

$$
\int_{0}^{\infty} t^{\mu-v+1} J_{\mu}(a t) J_{v}(b t) d t=0 \quad(0<b<a),
$$

and also that

$$
\int_{0}^{\infty} t^{-\lambda} J_{v-\lambda-1}(a t) J_{v}(b t) d t=\left\{\begin{array}{l}
0, \text { if } 0<b<a, \\
\frac{b^{-v} a^{v-\lambda-1}\left(b^{2}-a^{2}\right)^{\lambda}}{2^{\lambda} \Gamma(1+\lambda)}, \text { if } 0<a<b,
\end{array}\right\}
$$

provided that $v>\lambda>-1$; this implies, by the Hankel inversion theorem, that

$$
\int_{a}^{\infty} J_{v}(b t) b^{1-v}\left(b^{2}-a^{2}\right)^{\lambda} d b=2^{\lambda} \Gamma(1+\lambda) a^{1+\lambda-v} t^{-\lambda-1} J_{v-\lambda-1}(a t),
$$

provided that $\uparrow$

$$
v>2 \lambda+\frac{1}{2}>-\frac{3}{2} \text {. }
$$

On comparing (7) and (3), we see that a particular solution of (3) is the function $u^{\mu-v+1-a} J_{\mu}(u y)$, with $y>1, v>\mu>-1$ and so, by superposition, we are led to the expression

$$
\psi(u)=u^{\mu-v+1-\alpha} \int_{1}^{\infty} y \phi(y) J_{\mu}(u y) d y \quad(\mu>v>-1) .
$$

Substituting (12) into (3), inverting the order of integration and using (9), we verify that (12) satisfies (3).

But now the Hankel inversion theorem applied to (12) shows that

$$
\int_{0}^{\infty} u^{v+\alpha-\mu} \psi(u) J_{\mu}(u y) d u= \begin{cases}\phi(y), & \text { if } y>1, \\ 0, & \text { if } 0 \leqq y<1,\end{cases}
$$

i.e., if $\psi(u)$ is given by (12), then (3) may be replaced by the equivalent equation

$$
\int_{0}^{\infty} u^{v+\alpha-\mu} \psi(u) J_{\mu}(x u) d u=0 \quad(0 \leqq x<1)
$$

provided that $\nu>\mu>-1$.

$\uparrow$ This condition is discussed in $\$ 4$. 
To transform (4) we replace $x$ by $x y$, multiply both sides by $y^{1-v}\left(y^{2}-1\right)^{\lambda}$, integrate with respect to $y$ from 1 to $\infty$, and invert the order of integration on the left-hand side:

$$
\int_{0}^{\infty} \psi(u)\left\{\int_{1}^{\infty} J_{v}(x y u) y^{1-v}\left(y^{2}-1\right)^{\lambda} d y\right\} d u=\int_{1}^{\infty} g(x y) y^{1-v}\left(y^{2}-1\right)^{\lambda} d y
$$

hence, by (10),

$$
\int_{0}^{\infty} u^{-\lambda-1} \psi(u) J_{v-\lambda-1}(x u) d u=\frac{x^{\lambda+1}}{2^{\lambda} \Gamma(1+\lambda)} \int_{1}^{\infty} g(x y) y^{1-v}\left(y^{2}-1\right)^{\lambda} d y \quad(x>1),
$$

provided that $v>2 \lambda+\frac{1}{2}>-\frac{3}{2}$.

If now $\lambda$ and $\mu$ are chosen so that $v+\alpha-\mu=-\lambda-1$ and $\mu=v-\lambda-1$, i.e.

$$
\lambda=-1-\frac{1}{2} \alpha, \quad \mu=v+\frac{1}{2} \alpha
$$

(these values satisfy (8) and (11), provided that $\alpha<0,2 v+\alpha+2>0$ and $2 v+2 \alpha+3>0$ ), then (14) and (15) become

$$
\int_{0}^{\infty} u^{\frac{1}{2} \alpha} \psi(u) J_{v+\frac{1}{2} \alpha}(x u) d u=\left\{\begin{array}{l}
0 \text { if } 0 \leqq x<1, \\
\frac{x^{-\frac{1}{2} \alpha} 2^{1+\frac{1}{2} \alpha}}{\Gamma\left(-\frac{1}{2} \alpha\right)} \int_{1}^{\infty} g(x y) y^{1-v}\left(y^{2}-1\right)^{-1-\frac{1}{2} \alpha} d y \quad \text { if } x>1 .
\end{array}\right\}
$$

Hence, by the Hankel inversion theorem,

$$
\begin{gathered}
\psi(x)=\frac{x^{1-\frac{1}{2} \alpha} 2^{1+\frac{1}{2} \alpha}}{\Gamma\left(-\frac{1}{2} \alpha\right)} \int_{1}^{\infty} u^{1-\frac{1}{2} \alpha} J_{v+\frac{1}{2} \alpha}(x u)\left\{\int_{1}^{\infty} g(u y) y^{1-v}\left(y^{2}-1\right)^{-1-\frac{1}{2} \alpha} d y\right\} d u \\
=-\frac{x^{1-\frac{1}{2} \alpha} 2^{\frac{1}{2} \alpha}}{\Gamma\left(1-\frac{1}{2} \alpha\right)} \int_{1}^{\infty} u^{v+\frac{1}{2} \alpha} J_{v+\frac{1}{2} \alpha}(x u) \frac{d}{d u}\left\{\int_{u}^{\infty} g(v) v^{1-v}\left(v^{2}-u^{2}\right)^{-\frac{1}{1} \alpha} d v\right\} d u
\end{gathered}
$$

provided that $\alpha<0,2 v+\alpha+2>0$, and $2 v+2 \alpha+3>0$, where we have put $u y=v$ and written

$$
\left(v^{2}-u^{2}\right)^{-1-\frac{1}{2} \alpha}=-\left\{(2 u)\left(-\frac{1}{2} \alpha\right)\right\}^{-1} \frac{d}{d u}\left(v^{2}-u^{2}\right)^{-\frac{1}{2} a}
$$

3. To deal with the case $\alpha>0$, instead of choosing $\lambda$ and $\mu$ as in (16), we let

$$
\lambda=-\frac{1}{2} \alpha, \quad \mu=v+\frac{1}{2} \alpha-1 .
$$


These values satisfy (8) and (11) provided that $\alpha<2,2 v+\alpha>0$ and $2 v+2 \alpha-1>0$ and so this time (14) and (15) become, respectively,

$$
\begin{aligned}
\int_{0}^{\infty} u^{1+\frac{1}{2} \alpha} \psi(u) J_{v+\frac{1}{2}-1}(x u) d u=0 \quad(0 \leqq x<1), & \\
\int_{0}^{\infty} u^{\ddagger \alpha-1} \psi(u) J_{v+t a-1}(x u) d u & =\frac{x^{1-\frac{1}{2} \alpha} 2^{\frac{1}{a} a}}{\Gamma\left(1-\frac{1}{2} \alpha\right)} \int_{1}^{\infty} g(x y) y^{1-v}\left(y^{2}-1\right)^{-\frac{1}{2} a} d y \quad(x>1), \\
& =F(x), \text { say } \quad(x>1) .
\end{aligned}
$$

We now use the result (Watson $[10$, p. 18])

$$
\frac{d}{d x}\left\{x^{n} J_{n}(x y)\right\}=y x^{n} J_{n-1}(x y)
$$

to write $J_{v+\frac{1}{\alpha}-1}(x u)=u^{-1} x^{-v-\frac{1}{\alpha} \alpha} \frac{d}{d x}\left\{x^{v+\frac{1}{2} \alpha} J_{v+\frac{1}{2} \alpha}(x u)\right\}$ in (20). Integrating with respect to $x$ from 0 to $x$, we find (using the fact that $v+\frac{1}{2} \alpha>0$ ) that

$$
\int_{0}^{\infty} u^{\frac{1}{2} \psi} \psi(u) J_{v+\frac{\ddagger}{t a}}(x u) d u=0 \quad(0 \leqq x<1)
$$

Similarly, if we multiply (22) by $x^{-v-t a+1}$, differentiate with respect to $x$ and use the result (Watson [10, p. 18])

$$
\frac{d}{d x}\left\{x^{-n} J_{n}(x y)\right\}=-y x^{-n} J_{n+1}(x y)
$$

we find that

$$
\int_{0}^{\infty} u^{\ddagger a} \psi(u) J_{v+\frac{f a}{d a}}(x u) d u=-x^{v+\frac{1}{d a}-1} \frac{d}{d x}\left\{x^{-v-\frac{1}{2} a+1} F(x)\right\} \quad(x>1) .
$$

Combining (24) and (26) and using the Hankel inversion theorem, as before, we obtain

$$
\psi(x)=-x^{1-\frac{1}{2} a} \int_{1}^{\infty} u^{v+\frac{1}{2} \alpha} J_{v+\frac{1}{2} a}(x u) \frac{d}{d u}\left\{u^{-v-\frac{1}{d} \alpha+1} F(u)\right\} d u,
$$

which is easily seen to be identical with (18) if we use (22) and put $u y=v$ in the inner integral. Note that Gordon [3] gave the solution in different forms for the two cases $\alpha>0$ and $\alpha<0$.

Thus the solution of (3) and (4) is given by (18) in the two cases $-2<\alpha<0,2 v+\alpha+2>0$, $2 v+2 \alpha+3>0$ and $0<\alpha<2,2 v+\alpha>0,2 v+2 \alpha-1>0$. Therefore, if we combine (18) 
with Copson's form [7] of the solution of (1) and (2) in the case $g \equiv 0$, we obtain as the solution of (1) and (2)

$$
\begin{aligned}
& \psi(x)=\frac{x^{1-\frac{1}{2} \alpha} 2^{-\frac{1}{2} \alpha}}{\Gamma\left(1+\frac{1}{2} \alpha\right)} \int_{0}^{1} u^{-v-\frac{1}{2} \alpha} J_{v+\frac{1}{2} \alpha}(x u) \frac{d}{d u}\left\{\int_{0}^{u} f(v) v^{1+v}\left(u^{2}-v^{2}\right)^{\frac{1}{2} \alpha} d v\right\} d u \\
&--\frac{x^{1-\frac{1}{2} \alpha} 2^{\frac{1}{2} \alpha}}{\Gamma\left(1-\frac{1}{2} \alpha\right)} \int_{1}^{\infty} u^{v+\frac{1}{2} \alpha} J_{v+\frac{1}{2} \alpha}(x u) \frac{d}{d u}\left\{\int_{u}^{\infty} g(v) v^{1-v}\left(v^{2}-u^{2}\right)^{-\frac{1}{2} \alpha} d v\right\} d u
\end{aligned}
$$

under the conditions

$$
\left.\begin{array}{cll}
-2<\alpha<0, & 2 v+\alpha+2>0, & 2 v+2 \alpha+3>0, \\
0<\alpha<2, & 2 v+\alpha>0, & 2 v+2 \alpha-1>0 .
\end{array}\right\}
$$

4. It remains to discuss the restrictions on the parameters. Although equation (9) is valid under the condition $v>\lambda>-1$, equation (10) is valid under the more restrictive condition (11). The reason is that the Hankel inversion theorem (see, e.g., [10, p. 456])

$$
\begin{aligned}
& \bar{F}(x)=\int_{0}^{\infty} u F(u) J_{v}(x u) d u, \\
& F(x)=\int_{0}^{\infty} u \bar{F}(u) J_{v}(x u) d u,
\end{aligned}
$$

where

is valid if $F(y)$ is continuous at $y=x$ and $\int_{0}^{\infty} x^{\frac{1}{2}} F(x) d x$ is absolutely convergent. Applying the latter condition to the function $F(t)=t^{-\lambda-1} J_{v-\lambda-1}(a t)$, we find (see [11, pp. 71-72]) that (10) is valid under the condition $v>2 \lambda+\frac{1}{2}>\frac{1}{2}$. However, direct examination shows that the left-hand side of (10) converges when $v>2 \lambda+\frac{1}{2}>-\frac{3}{2}$ and therefore, by analytic continuation, (10), and so also (15), is valid under the condition (11).

It should be noted that if (11) is replaced by the condition $v>\lambda>-1$, then the conditions for the validity of the solution turn out to be $0<\alpha<2,2 v+\alpha>0$ and $-2<\alpha<0$, $2 v+\alpha+2>0$, i.e. precisely the conditions obtained by other methods (cf. [4], [6], [7]; this point is discussed more fully in [4]). These conditions are less restrictive than the conditions (29) when $-2<\alpha<-1$ and when $0<\alpha<1$.

This suggests that in fact (15) is valid under the less restrictive condition $v>\lambda>-1$, rather than (11), and raises the question of some other method of derivation, one avoiding the Hankel inversion theorem. One such method is the use of Parseval Theorem (see [9, p. 59]):

$$
\int_{0}^{\infty} x F(x) G(x) d x=\int_{0}^{\infty} u \bar{F}(u) \bar{G}(u) d u
$$

provided that $\int_{0}^{\infty} x^{\frac{1}{2}} F(x) d x$ and $\int_{0}^{\infty} x^{\frac{1}{2}} G(x) d x$ are absolutely convergent, where $\bar{F}$ and $\bar{G}$ are the Hankel transforms of $F(x)$ and $G(x)$, respectively, given by (30). Applying (32) to (4) and (9), we easily find (15); but with $F(t)=t^{-\lambda-1} J_{v-\lambda-1}(a t)$ the condition of absolute convergence 
yields the condition $v>2 \lambda+\frac{1}{2}>\frac{1}{2}$, already mentioned, which is even more restrictive than (11).

It appears that, without a detailed discussion of the convergence of the integrals (as was carried out by Busbridge [2]), the method presented above† is simply a formal procedure for obtaining the solution which cannot be relied upon to furnish the conditions for validity of this solution. The same is true of Gordon's original method [3], where the validity of a Hankel inversion does not come into question until it applies to an expression involving an unknown function (as in going from (17) above to (18)), and also of other methods of solution (as was suggested in [7]). In all these methods a source of difficulty is the fact that conditions for the order of integrations, or of integration and differentiation, to be inverted are assumed to be satisfied (as was done above) but these conditions are not reflected in restrictions on the parameters.

† Related ideas have recently been used by Peters [12] and Sneddon [13].

\section{REFERENCES}

1. E. C. Titchmarsh, Introduction to the theory of Fourier integrals (Oxford, 1937), 334-339.

2. I. W. Busbridge, Dual integral equations, Proc. London Math. Soc. (2) 44 (1938), 115-129.

3. A. N. Gordon, Dual integral equations, J. London Math. Soc. 29 (1954), 360-363.

4. J. Burlak, On the solution of certain dual integral equations, Proc. Glasgow Math. Assoc. 6 (1963), 39-44.

5. B. Noble, On some dual integral equations, Quart. J. Math. Oxford Ser. (2) 6 (1955), 81-87.

6. M. Lowengrub and I. N. Sneddon, On certain dual integral equations, U.S. Air Force Technical Report, Contract No. AF 49(638)-892, 1961. [See also: The solution of a pair of dual integral equations, Proc. Glasgow Math. Assoc. 6 (1963), 14-18.]

7. E. T. Copson, On certain dual integral equations, Proc. Glasgow Math. Assoc. 5 (1961), 21-24.

8. B. Noble, Certain dual integral equations, J. Math. and Phys. 37 (1958), 128-136.

9. I. N. Sneddon, Fourier Transforms (New York, 1951), p. 52.

10. G. N. Watson, A treatise on the theory of Bessel functions (Cambridge, 2nd edition, 1944).

11. E. T. Whittaker and G. N. Watson, $A$ course of modern analysis (Cambridge, 4th edition, 1927).

12. A. S. Peters, Certain dual integral equations and Sonine integrals (New York University, Research Report IMM-NYU 285, August 1961).

13. I. N. Sneddon, Fractional integration and dual integral equations (North Carolina State College, June 1962).

North Carolina State College

Raleigh, North Carolina, U.S.A.

(Permanent address: The University, Glasgow, W. 2) 